\title{
The Origin of the Vertebrate Eye
}

\author{
Trevor D. Lamb • Edward N. Pugh Jr. • Shaun P. Collin
}

Published online: 30 September 2008

(C) Springer Science + Business Media, LLC 2008

\begin{abstract}
In his considerations of "organs of extreme perfection," Charles Darwin described the evidence that would be necessary to support the evolutionary origin of the eye, namely, demonstration of the existence of "numerous gradations" from the most primitive eye to the most perfect one, where each such tiny change had provided a survival advantage (however slight) to the organism possessing the subtly altered form. In this paper, we discuss evidence indicating that the vertebrate eye did indeed evolve through numerous subtle changes. The great majority of the gradual transitions that did occur have not been preserved to the present time, either in the fossil record or in extant species; yet clear evidence of their occurrence remains. We discuss the remarkable "eye" of the hagfish, which has features intermediate between a simple light detector and an image-forming camera-like eye and which may represent a step in the evolution of our eye that can now be studied by modern methods. We also describe the important clues to the evolutionary origin of the vertebrate eye that can be found by studying the embryo-
\end{abstract}

T. D. Lamb $(\bowtie)$

ARC Centre of Excellence in Vision Science, and Division of Neuroscience, John Curtin School of Medical Research, The Australian National University,

Canberra, ACT 0200, Australia

e-mail: Trevor.Lamb@anu.edu.au

E. N. Pugh Jr.

F.M. Kirby Center for Molecular Ophthalmology, Stellar-Chance Building, University of Pennsylvania,

Philadelphia, PA 19104-6069, USA

S. P. Collin

School of Biomedical Sciences, The University of Queensland, Brisbane, Queensland 4072, Australia logical development of our own eye, by examining the molecular genetic record preserved in our own genes and in the genes of other vertebrates, and through consideration of the imperfections (or evolutionary "scars") in the construction of our eye. Taking these findings together, it is possible to discuss in some detail how the vertebrate eye evolved.

Keywords Evolution $\cdot$ Vertebrate eye $\cdot$ Retina Photoreceptors · Opsins

\section{Introduction}

As we shall discuss, there is now overwhelming evidence that the vertebrate eye did indeed arise through an evolutionary sequence involving countless tiny steps. However, a full picture of the historical sequence remains hidden from our view for two major reasons. Firstly, the most important advances in the organization of what would eventually become the vertebrate eye occurred over 500 million years ago (Mya), prior to the evolution of hard body parts (like a bony skeleton), and as a result, many such advances in the arrangement of the vertebrate eye occurred in animals that are either not preserved, or else are very poorly represented in the fossil record. Secondly, each of those eye arrangements that was superseded by a better arrangement is very unlikely to have survived for hundreds of millions of years in the face of competition from animals possessing better eyes, and as a result, very few extant species retain eyes with the intermediate features. Nevertheless, several extant organisms do appear to retain eyes that provide remarkable windows into the sequence of events that took place. In addition, the genes of vertebrates retain detailed clues about their origins, and modern phylogenetic approaches can help piece together evolution- 
ary sequences. Likewise, the sequence of events occurring during embryonic development can, with careful interpretation, provide information about events that are likely to have occurred during evolution. And finally, certain apparent imperfections in the structure of the eye provide major clues to the evolutionary events that took place.

In order to assist the reader in appreciating the relevance and impact of these widely different approaches, we begin by summarizing the scenario that we have recently proposed for the evolution of the vertebrate eye. Although there is substantial and convincing experimental support for the picture that we paint below, it needs to be borne in mind that because of the imperfect nature of the record, the precise sequence of intermediate steps that we propose may need to be revised in the future.

\section{Scenario for the Steps Underlying the Evolution of the Vertebrate Eye}

Figure 1 presents a scenario for the likely evolution of the vertebrate eye, based on the proposals set out in our recent review (Lamb et al. 2007). This diagram will serve as the basis for our presentation of the evidence supporting the view that the eye did indeed evolve through numerous stages. The chronology set out in Fig. 1 is derived primarily from the fossil record.

\section{Primordial Photoreceptors and Photopigments}

As long as 580 Mya (right side of Fig. 1), at the stage when our own line, the deuterostomes, split from protostomes (most invertebrates), the common ancestors of those primitive organisms already possessed photoreceptors (i.e., light-sensitive cells) that presumably mediated functions such as phototaxis and circadian rhythms. However, "imaging vision" (providing a directional view of the world) had not yet evolved; thus, the photoreceptors were not associated with any kind of image-forming apparatus, and in any case, the organisms did not possess a sufficiently complex nervous system to process visual signals, had they been available. Two kinds of photoreceptor already existed (Fig. 1a): rhabdomeric photoreceptors, which became the dominant type in most invertebrates, and ciliary photoreceptors, which became the dominant type in vertebrates. In the past decade, it has become clear that protostomes and deuterostomes inherited both types of photoreceptor, and there are many examples of extant species in which rhabdomeric and ciliary photoreceptors coexist.

Ciliary and rhabdomeric photoreceptors exhibit substantial differences, perhaps most obviously in terms of the membrane expansion in which the photopigment, rhodopsin, is incorporated: In rhabdomeric photoreceptors, this
Fig. 1 Scenario for evolution of the vertebrate eye. Left Ciliary and rhabdomeric photoreceptors. a Prior to the divergence of protostomes and deuterostomes, more than 580 Mya, the primitive bilateral organisms possessed both ciliary and rhabdomeric photoreceptors. b At an early stage during deuterostome evolution, it seems likely that the rhabdomeric photoreceptors received synaptic input from the ciliary photoreceptors, which then no longer projected centrally. c Subsequently, the rhabdomeric photoreceptors lost their photoreceptive structures and became simply projection neurons (retinal ganglion cell, $R G C$ ). The ciliary photoreceptors required contact with RPE cells in order to re-synthesize the correct isomer of vitamin A needed by the visual pigment, opsin; this was achieved by the inward folding of the eye vesicle to form an eye-cup (see $f$ in middle column). Middle Presumed evolution of the neural tube, eye vesicle, eye-cup, and lens, based on the embryological development of the nervous system of extant vertebrates. $a-d$ Early in the evolution of bilateral animals, an inward folding of neural tissue occurred, which created the neural tube in chordates (by $550 \mathrm{Mya}$ ); the orange shading represents the lips of the neural folds. Light-sensitive cells were located in the regions that bulged laterally to form the "eye vesicles" (dashed box in e). Contact between the expanding neural tube and the outer layer of the animal (the surface ectoderm) triggered an in-folding of the optic vesicle to form the invaginated eye-cup $(f)$, in which the retina is apposed to the RPE. We propose that the eye-cup stage illustrated in $f$ had evolved prior to the divergence of myxinoids (as represented by extant hagfish) from our own line. Subsequently, in the line that led to both jawless and jawed vertebrates, the outer layer (ectoderm) thickened and gave rise to the formation of a lens $(g)$; this stage had arisen by the time of the last common ancestor that we shared with lampreys, about 500 Mya. Right Approximate time-line for the progressions sketched on the left and in the middle, with times indicated in millions of years ago (Mya) [Middle panel from Lamb et al. (2007) Fig. 4; first published in Nature Reviews Neuroscience 8, 2007 (C) Nature Publishing Group, a division of Macmillan Publishers Limited]

membrane takes the form of microvilli, whereas in ciliary photoreceptors it takes the form of out-foldings from a modified cilium. On the other hand, rhabdomeric and ciliary photoreceptors share many fundamental features, and they undoubtedly have a common origin (Arendt and Wittbrodt 2001; Arendt 2003). Their photopigments clearly arose from a common ancestor, and both represent specialized forms of a so-called GPCR, or G-protein coupled receptor, that is used widely in numerous other sensory and cellular signaling pathways.

\section{Neurulation and Lateral Expansion of the Developing Nervous System Forms Eye Vesicles}

Within 30 million years (i.e. by about $550 \mathrm{Mya}$ ), chordates had evolved. In these organisms, a process called neurulation occurs (Fig. 1a-d), whereby dorsal embryonic neural tissue folds upwards and inwards to meet, thereby forming a "neural tube," which gives rise to the dorsal nerve cord and the brain. The interior corresponds to the cerebral vesicles and contains cerebrospinal fluid (CSF), while the wall contains neuronal cells, including a variety of sensory receptor cells. We postulate that, by this stage, the ciliary photoreceptors were located at the inner surface (in contact with CSF), whereas the rhabdomeric photoreceptors were 


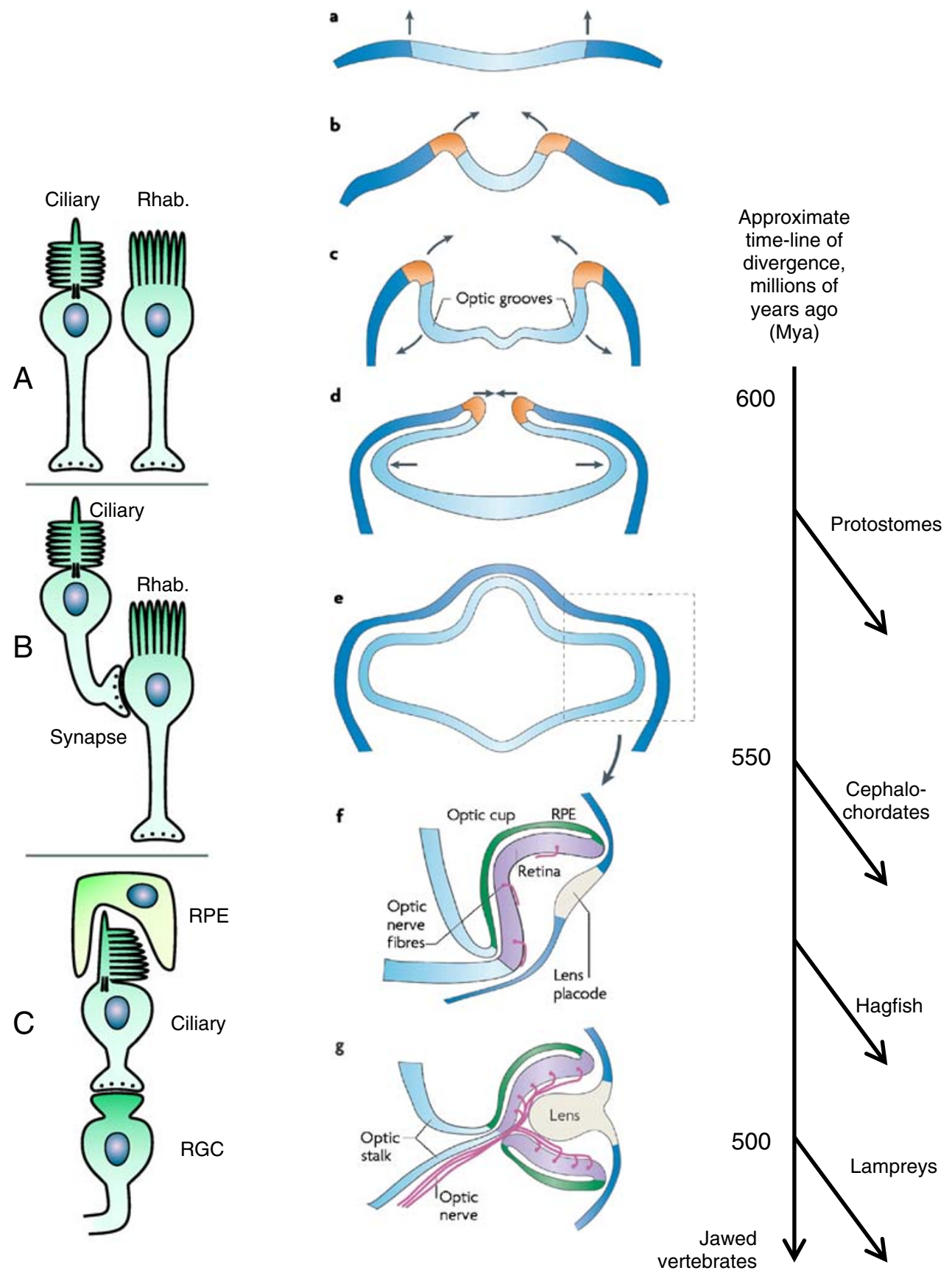

located at the outer surface. They became projection neurons which sent axons across the outer surface to make contact with diencephalic brain regions such as the hypothalamus.

An arrangement of this kind may have been entirely adequate for signaling daytime light levels in animals that had relatively transparent bodies and that inhabited shallow seas. However, it would have presented problems for animals attempting to colonize deeper waters, where the light levels would have been much lower. In such situations, the animal's photosensitivity could have been increased simply by increasing the number of photoreceptors, and that could have been accomplished most effectively by expansion of the photosensitive region. For craniates (animals with skulls), there may have been a distinct advantage if such expansion occurred by lateral ballooning (Fig. 1c-e), so that the light-sensitive region was not shadowed by the protective cranium that developed around the central nervous system.

At this stage of evolution, an inherent factor associated with ciliary rhodopsins probably came in to play. Whereas rhabdomeric rhodopsins are able to reconvert the "used" form (metarhodopsin) back into the "receptive" form simply by absorbing another photon of light, this trick is not used by ciliary rhodopsins. As a result, ciliary photo- 
receptors are dependent on a supply of a particular form of vitamin A (11-cis retinaldehyde), which has to be synthesized by a complicated biochemical pathway (see Lamb and Pugh 2004). In simple chordates, this was (and still is) accomplished by interspersing photoreceptors and one or more retinoid-synthesizing cells within the same layer; typically, these latter cells are pigmented and absorb stray light.

If part of the driving force for the ballooning of the photosensitive region was indeed the capture of a higher proportion of the incident light at very low intensities, then it would have been disadvantageous to continue to cover a substantial fraction of the absorbing area with nontransducing cells. If, instead, the pigmented cells could have been discarded from the photoreceptive layer and placed behind it, then the detection of light would have been improved because the entire layer would have comprised photoreceptor cells. It appears that this course was adopted in early craniates by causing the ballooning eye vesicle to fold inwards upon itself (Fig. 1e-f). The signal triggering this invagination seems almost certain to have arisen from the outer body wall (surface ectoderm) at a specialized region called a placode. Even though this region is given the name "lens placode" in modern vertebrates, our view is that its original function was likely to have been in triggering invagination of the eye vesicle at a stage in evolution prior to the appearance of a lens.

Further expansion of the light-sensitive region then resulted in the "eye-cup" illustrated in Fig. 1g. The retina wrapped around the axons that make up the optic nerve so that the optic nerve has a natural exit from the inner surface of the retina. Our hypothesis is that the "eye" of extant hagfish (see below) remains relatively unchanged from the state that it had reached more than 500 Mya. Thus, we think that in the particular ecological niche occupied by hagfish, there was no advantage to be gained by converting this non-visual organ into an imaging one.

However, for the organisms that subsequently became vertebrates, it seems that the tissue of the placode must have thickened slightly, thereby bending incoming light rays and giving rise to a weak optical lens, providing some slight degree of imaging onto the retina. Provided that the retina was able to process such an image and to send this information to an appropriate region of the primitive brain, then the animal would have possessed a rudimentary ability to resolve spatial images; that is, a simple form of "vision" would have existed. It is not hard to imagine that from this stage onwards, further improvements (Fig. 1g) would have snowballed with enormous speed because of the great survival advantage conveyed by any improvement in the visual ability of an organism inhabiting a world where other creatures had poorer (or non-existent) vision.

During an intense "burst" of evolution, the momentum of this tumultuous change in visual ability culminated in a remarkable period known as the Cambrian Explosion. Major advances in body plan appeared in a wide variety of organisms, and it is possible that one of the driving forces in this explosion was the emergence of vision. In the chordate line, animals possessing very rudimentary lightsensing organs evolved into early vertebrates possessing sophisticated eyes quite similar to our own. This rapid period of vertebrate eye evolution occurred over an interval possibly as short as 30 million years, with the modern vertebrate camera-style eye having evolved roughly 500 Mya (by the time that the predecessors of lampreys diverged from the lineage that gave rise to jawed vertebrates including humans). Further refinements to the form of the vertebrate eye have, of course, continued to evolve through to the present day, better adapting the vision of modern species to their distinct photic environments and visual behaviors. However, it seems that the fundamental pattern was already so exquisite by the time jawed vertebrates first appeared that relatively little change has taken place in the form of the vertebrate eye during the last 400 million years.

\section{Evidence Supporting this Evolutionary Scenario}

\section{Emergence of the Vertebrate-Style Eye: Evidence From Lampreys}

In order to establish just how far back in evolutionary history the vertebrate-style eye emerged, one can look for our most distant relative that shares a similar type of eye. Remarkably, one finds that all jawed vertebrates possess eyes so closely similar to our own that it is indisputable that the last common ancestor of jawed vertebrates (that lived around 430 Mya) was equipped with an eye fundamentally the same as ours. But is it possible to go back even further?

The ancestors of modern lampreys are thought to have diverged from our own (jawed vertebrate) line about 500 Mya (Fig. 1). Yet the eyes of adult lampreys are surprisingly similar to those of all jawed vertebrates. Like our eye, the lamprey's eye is camera-style, with a lens, an iris, and extra-ocular muscles. Its retina is also very similar to ours, with three distinct layers of cell nuclei, comprising the cell bodies of photoreceptors, interneurons, and output neurons (ganglion cells). Furthermore, its photoreceptors and its photopigments (opsins) are also closely similar to those of jawed vertebrates; the southern hemisphere lamprey, Geotria australis, has five classes of cone-like photoreceptor, each of which contains an opsin broadly corresponding to each of the five classes of jawedvertebrate opsins. The overall similarities are so extensive that convergent evolution can be ruled out, and the conclusion seems inescapable that a vertebrate-style eye 
must have been present in the last common ancestor of lampreys and jawed vertebrates, around 500 Mya.

Are there any extant animals that diverged from our line even earlier, yet have a "vertebrate-style" eye? Yes and no. As we discuss next, the hagfish possesses what appears to be a forerunner to the vertebrate eye, though we think it somewhat misleading to elevate this organ to the category of an "eye."

Hagfish: Living Descendants of a "Missing Link" in the Evolution of the Vertebrate Eye?

In our view, hagfish provide important clues to the evolution of the vertebrate eye. Hagfish (Fig. 2a) are a group of primitive, jawless, eel-shaped, marine chordates, characterized by the copious amounts of gelatinous slime that their skin exudes when they are threatened (hence their alternate name of slime eels). They are widespread throughout the world's oceans, though most are restricted to great depths (200-1000 m), where they feed on carcasses of dead animals that have fallen to the seabed. Of extant craniates (animals with skulls), hagfish have the most basal body plan; they possess neither jaws nor vertebrae, and they are usually regarded not as vertebrates but rather as a sister group.
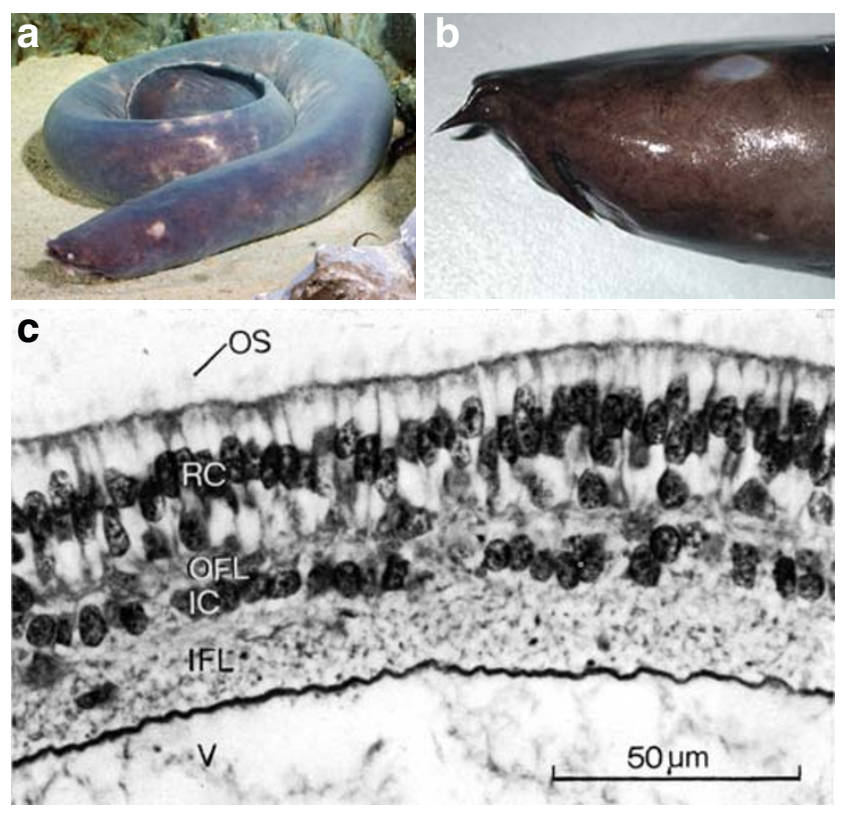

Fig. 2 The hagfish and its retina. a The New Zealand hagfish, Eptatretus cirrhatus. b The head region of the same species. In a and $\mathbf{b}$, the prominent pale patch is the translucent skin beneath which the hagfish "eye" is buried. c The retina of the hagfish, Eptatretus stouti, viewed using light microscopy; $O S$ outer segments; $R C$ receptor cells; $O F L$ outer fiber layer (=outer plexiform layer); $I C$ inner cells (=retinal ganglion cells); IFL inner fiber layer; $V$ vitreous. The images are reproduced with permission as follows: a Dr Paddy Ryan CRyan Photographic; b CAustralian Museum, photo Mark McGrouther; c from Holmberg (1977), reproduced by permission of Springer
The phylogenetic relationship of hagfish to lampreys and jawed vertebrates is controversial; in other words, it is not entirely clear when the ancestors of hagfish, lampreys, and jawed vertebrates separated from each other. One view is that hagfish and lampreys are monophyletic, meaning that their lines did not diverge from each other until after their common ancestor had already diverged from our line. A second view (to which we subscribe) is that the ancestors of hagfish diverged from the vertebrate line prior to the appearance of the last common ancestor of lampreys and jawed vertebrates. Until the true phylogenetic position of hagfish is established with certainty, there will remain difficulties in interpreting the clues provided by their eyes.

Behaviorally, hagfish appear to be blind, and what little response they have to light seems to be mediated by photoreceptors in the skin. They appear to locate food using their keen sense of smell (olfaction), which is highly developed and to which a large proportion of their brain is devoted.

\section{The Hagfish Eye}

Hagfish possess a bilateral pair of eyes, though their "eye" is very different from ours. It is small, conical in shape, and completely lacking in any sign of a lens, a cornea, an iris, or intra- or extraocular muscles, and is buried beneath a patch of translucent skin (Fig. 2b); reviewed in Locket and Jorgensen (1998). Its retina (Fig. 2c) has a rudimentary appearance, comprising only two main nuclear layers containing the photoreceptors and the output neurons (retinal ganglion cells), respectively. In contrast to the vertebrate retina, there do not appear to be any intermediate neurons linking the photoreceptors to the output neurons, and instead, the photoreceptors are thought to synapse directly onto the output neurons. These output neurons project primarily to the hypothalamus, a part of the brain devoted to circadian and other hormonal functions. In contrast, the great majority of retinal ganglion cells in vertebrates project to "higher" regions of the brain, namely, to the optic tectum in non-mammals or in mammals to the thalamus, a sensory relay station en route to the visual cortex. The photoreceptors of the hagfish retina are homologous to those of the vertebrate retina, though they have a simpler structure (see below). Hence, in many respects, the hagfish eye more closely resembles the pineal organ of non-mammalian vertebrates than it does the vertebrate eye. Indeed, our view is that the hagfish "eye" does not subserve a visual role but is instead likely to function as a circadian organ, as does the pineal organ.

It is often suggested that the hagfish eye is degenerate, having regressed from a more lamprey-like eye that existed in the common ancestor of hagfish and lampreys. We find this view implausible. Given that the hagfish eye has 
survived for hundreds of millions of years, in comparison with the degeneration that has occurred in just thousands of years in cavefish, it seems clear that the organ must have been of considerable value to the survival of the hagfish. On the degeneration hypothesis, one would need to explain how it was advantageous to hagfish for the following to have occurred: (a) for a three-layered retina to have reverted to a two-layered structure; (b) for the processing power of the bipolar cells to have been dispensed with; (c) for a reversion to a more rudimentary photoreceptor structure; (d) for the disappearance of the lens, cornea, iris, and ocular muscles, all without trace; (e) for the covering of the eye by skin; and (f) for the projection of the retinal ganglion cells to be redirected from the tectum primarily to the hypothalamus. If the eye had been of no real value to hagfish, then rather than becoming transformed in these ways, it seems more likely (or more parsimonious in evolutionary terms) that it would simply have been lost over the extremely long interval of several hundred million years.

Our view, instead, is that this organ has always been important to the hagfish, but as a circadian organ. Due to the particular ecological niche that the hagfish has occupied at the bottom of the ocean, there was never any advantage to be gained from chance modifications that provided any degree of optical imaging or any increase in retinal computational power. Therefore, the hagfish has simply retained a circadian organ, capable of sensing the light levels that are available in deep water, with little change over geological time. It is important to realize that even though hagfish may seem "primitive," they have been subject to over 500 million years of potential evolutionary pressure since they shared a common ancestor with us. Nevertheless, we suggest that the hagfish "eye" may not have changed in a major way during that time, in part because the animals developed a protective mechanism (sliming), and occupied an ecological niche, which protected them from the fiercest competition. As a result, the hagfish "eye" may provide a window into an evolutionary step in the emergence of our own eye.

\section{Metamorphosis of the Larval Lamprey Eye}

A remarkable process of development takes place in the eyes of lampreys, which is consistent with the scenario outlined in Fig. 1. The larval form of the lamprey (called an ammocoete) develops slowly, over a period of 5 years or more, before metamorphosing into the adult (Fig. 3). The ammocoete is effectively blind (though it may detect light), and its paired eyes are similar to those of the hagfish in that they are small and buried beneath skin. The retina appears to be relatively undifferentiated (neuroblastic), and over a period of several years, it slowly differentiates in a sequence comparable to that which occurs far more rapidly
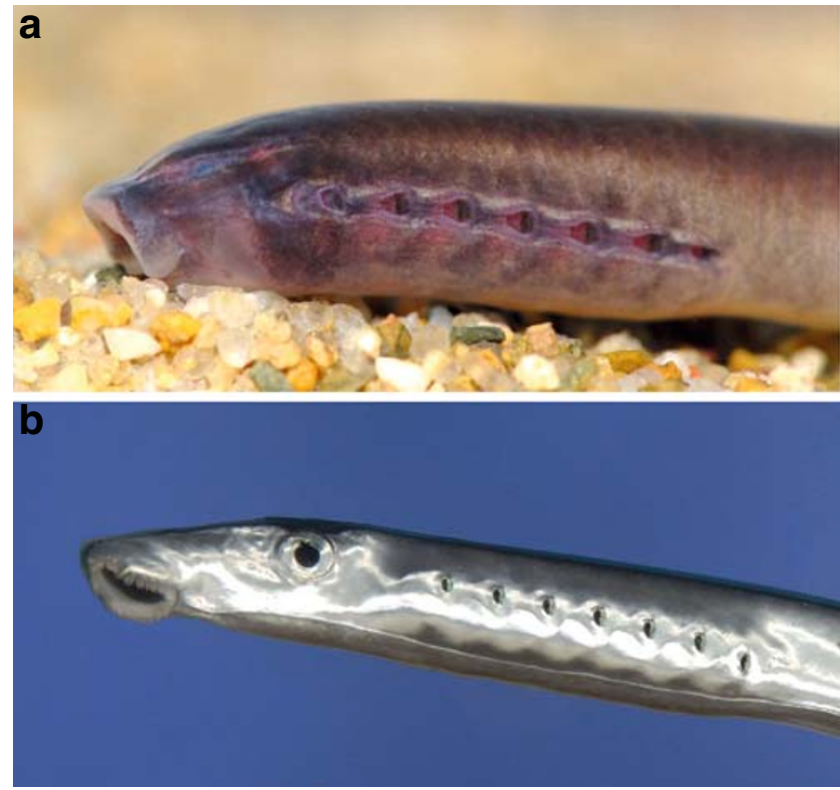

Fig. 3 Southern hemisphere lamprey, Geotria australis. a Larval form, "ammocoete". b Post-metamorphic, "downstream" adult form. Note that the ammocoete has the mouth and seven gill slits but that there is no sign of an eye at the surface. Instead, there is a rudimentary and small eye-like organ buried deep beneath the skin. In this and several other regards, the ammocoete resembles the hagfish. Photographs courtesy of G. Westhoff and S.P. Collin

in our own retina (see below). Then, at metamorphosis, the lamprey eye changes substantially: the retina differentiates fully; the lens, cornea, and extraocular muscles develop; and the eye enlarges and erupts at the surface in the form of a vertebrate-style eye. In other words, it is as if metamorphosis in the lamprey converts a hagfish-like non-visual organ into a vertebrate-like image-forming eye. We suggest that this process of metamorphosis reflects evolutionary developments that occurred over some 30 million years, as indicated in Fig. 1f $-\mathrm{g}$. Furthermore, we suggest that an analogous process occurs at a very much faster rate in the development of the retina of jawed vertebrates, as we discuss now.

\section{Development of the Mammalian Eye-Cup}

The developmental sequence that occurs during the formation of our own eye bears a close similarity to the scenario that we have put forward in Fig. 1 for the evolution of the eye. Alternatively, one may say that we have proposed our scenario so as to be broadly consistent with the events that occur during development. Hence, our proposals conform with the general notion put forward in the nineteenth century that the developmental stages through which an embryo passes bear a resemblance to the evolutionary history of that organism. This was eloquently stated by Darwin, who wrote, "Thus the embryo 
comes to be left as a sort of picture, preserved by nature, of the ancient and less modified condition of each animal" (Darwin 1859, p. 338). For an account of the way that such ideas arose from the experimental work of the embryologist von Baer (1828), see Richards (2009). Provided that this general notion is treated with caution, it can provide extremely useful heuristic insights into evolution. However, it is widely agreed that the subsequent restriction of this concept by Haeckel (1868) to the catchphrase "ontogeny recapitulates phylogeny" represents an oversimplification.

In the mammalian eye, the developmental sequence can be summarized as follows. In the early embryo, the neural tube forms, as the result of an upward and inward folding of neural tissue along much of the length of the embryo (corresponding to the evolutionary stages suggested in Fig. 1a-c). Towards the rostral (head) end of the embryo, the neural tube bulges outwards (Fig. 1c-e), with the ballooning regions being termed optic vesicles (Fig. 1e). Upon contact between the ballooning optic vesicle and the overlying surface of the embryo (termed the ectoderm), the eye vesicle is induced to fold inwards upon itself (Fig. 1f), bringing the developing retina into contact with the developing retinal pigment epithelium (RPE). At the same time, the eye-cup is expanding and growing around the developing optic nerve, eventually sealing over at the lower (ventral) margin (Fig. 1g), closing off a gap termed the choroidal fissure. From the stage at which the ballooning optic vesicle contacts the ectoderm (Fig. 1e), a parallel development occurs in the ectoderm: the ectoderm thickens and bulges inwards, eventually forming the lens (Fig. 1g). In other words, during mammalian embryogenesis, the development of the lens takes place in parallel with the invagination of the retina to form the eye-cup, whereas we have proposed that during evolution, the formation of a lens did not occur until after formation of the eye-cup. If we are correct, then this represents another departure from a literal interpretation of Haeckel's dogma.

\section{Development of Neural Processing Power \\ in the Mammalian Retina: Evidence for Evolution at a Cellular Level}

Evidence for evolution at a cellular level is not often documented, but as we now discuss, examination of the early development of the mammalian retina provides a compelling case for the way in which a simple retina containing only two cell classes evolved into the complex neural system that exists in all modern vertebrates. We describe the development and the presumed evolution of the retina's neural wiring, as well as the likely origin of an additional neural element, the retinal bipolar cell. Together, these advances provided a massive increase in the retina's computational power.
Neural Wiring The development of neural wiring in the mammalian retina provides support for our notion of an evolutionary progression from a hagfish-like retina to a lamprey-like retina. As indicated in Fig. 4a, immature photoreceptors (both cones and rods) initially make synaptic contacts with the output layer of the developing retina at the inner plexiform layer (Johnson et al. 1999). Although it is not yet clear whether these initial contacts are made onto amacrine cells (interneurons) or onto ganglion cells (output neurons), the situation is reminiscent of that in the hagfish, with just two principal cell layers involved in the wiring. Subsequently, two additional classes of retinal neuron (horizontal cells and bipolar cells) mature in sequence, and the photoreceptors retract their initial processes from the output layer and instead, make new synaptic contacts with the developing horizontal cells at an intermediate processing layer called the outer plexiform layer (Fig. 4b). The developing bipolar cells then insert themselves into the neural pathways, linking the photoreceptors to the output layer and thereby creating the adult wiring pattern (Fig. 4b). At an even later stage, the photoreceptor outer segments develop and mature (Fig. 4c).

This sequence, of the formation by photoreceptors of transient processes through to the inner retina, their retraction, and the formation of new contacts onto bipolar cells, is difficult to rationalize unless one has in mind the evolutionary sequence that is proposed in the scenario of Fig. 1 for the transformation of a hagfish-like two-layered retina (Fig. 3c) into a vertebrate three-layered retina (Fig. 4c). The situation represents yet another example of the maxim that "Nothing in biology makes sense except in the light of evolution" (Dobzhansky 1973).

Origin of Bipolar Cells A number of characteristic features of retinal bipolar cells provide clues to their likely origin. Bipolar cells are remarkably similar to photoreceptors in many regards. Even though they do not have a light-sensitive outer segment with membranes containing photopigment, many of them nevertheless possess a homologous ciliary structure called a Landolt club, the purpose of which remains obscure. In addition, many of the signaling proteins that they contain are either identical to, or else close homologues of, proteins in the photoreceptors. Furthermore, the bipolar cell's output terminal utilizes a specialized synaptic structure, called a ribbon synapse, that is almost identical to that in the photoreceptor, yet which is found nowhere else in the retina (and, outside of the retina, is found only in mechanosensitive hair cells, which are also modified cilia). Finally, during early retinal development, it is quite difficult to distinguish immature photoreceptors from immature bipolar cells because they have such similar bipolar morphology. One difference, though, is that the bipolar cells are born and mature at a slightly later stage of retinal development. 

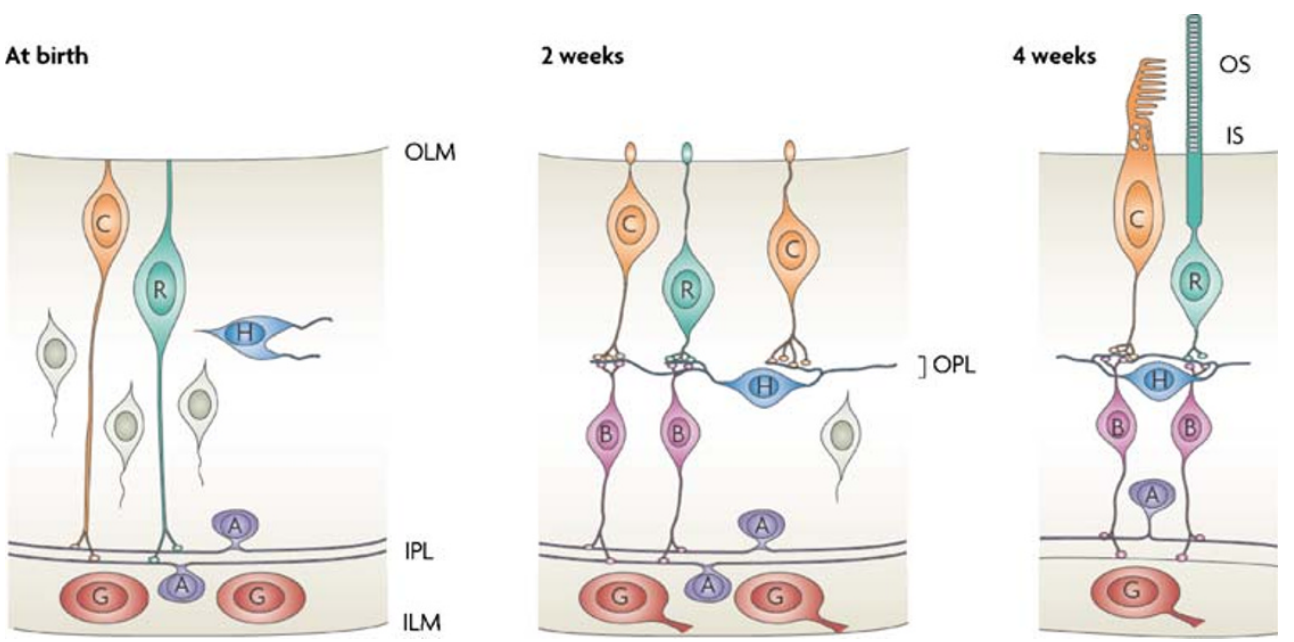

Fig. 4 Development of circuitry in the mammalian retina. Three stages of embryonic development observed in the ferret retina, as reported by Johnson et al. (1999). The ferret is particularly convenient because the young are born at a very immature stage, and the eyes do not open until 2 weeks after birth (middle panel). Left panel At birth, the cone and rod photoreceptors $(C$ and $R$ ) are not identifiable on the basis of morphology and display a simple bipolar shape; however, they are identifiable by certain molecular markers, such as rhodopsin. Very interestingly, the photoreceptors send processes (downwards in this diagram) directly to the retina's output layer, the so-called inner plexiform layer (IPL); however, it has not yet been established whether the neurons they contact are amacrine cells $(A)$ or ganglion cells $(G)$. At this stage, the horizontal cells $(H)$ and bipolar cells are barely

These features are consistent with the notion that retinal bipolar cells arose as some kind of a duplication event: perhaps as a duplication or a migration of a subset of photoreceptors, or perhaps as a duplication of a more primitive ciliated sensory cell. The advantage that their presence conveyed was an increase in neural computing power. Retinal bipolar cells permit the spatial pooling (or convergence) of signals from numerous photoreceptors onto ganglion cells, thereby increasing sensitivity. In addition, they initiate the push-pull arrangement of retinal signal processing; thus, bipolar cells come in two main varieties according to the polarity of the signal they generate, and provide the beginning of the "on" and "off" divisions of retinal signaling. It seems plausible to think that the ability to signal both a dark shadow and a light patch, via an increased firing rate in different classes of ganglion cell, together with the ability to pool signals over large areas, endowed the organism with a significant survival advantage.

\section{Darwin's "Numerous Gradations"}

In seeking evidence for the "numerous gradations" that Darwin anticipated must have existed at some stage during the evolution of our eye, it is important to realize that at the differentiated. Middle panel After a further 2 weeks, by the time of eye opening, the horizontal cells and bipolar cells $(B)$ have matured, and the photoreceptors now retract the processes that they had previously sent to the IPL and instead make new contacts with the horizontal and bipolar cells; this layer of synaptic connections forms the outer plexiform layer $(O P L)$ of the adult. Right panel At an even later stage of development, the photoreceptors extend an inner segment (IS) and a light-sensitive outer segment $(O S)$; it is not until this stage that conventional visual transduction becomes possible [from Lamb et al. (2007) Fig. 5; first published in Nature Reviews Neuroscience 8, 2007 (C) Nature Publishing Group, a division of Macmillan Publishers Limited]

level of whole organisms and whole eyes, the overwhelming majority will have been obliterated over time by the success of organisms that evolved further improvements. In other words, we do not expect to find examples of extant organisms retaining transitional forms of eyes corresponding to all the evolutionary steps that led up to the vertebrate eye; organisms with intermediate forms of eye configuration are unlikely to have been able to compete effectively against other organisms with improved eyes. What then accounts for the continued existence, over hundreds of millions of years, of the apparently primitive hagfish eye? In our view, it is the fact that it is not an eye at all, but instead a circadian organ that has survived in much the same way as the pineal organ of non-mammalian vertebrates has survived because it is fully functional and provides a selective advantage exactly as it is. Additional factors may be that hagfish have to some extent been protected from predation by the defense mechanism that they have developed and by the ecological niche that they have occupied.

Is it likely that transitional forms of eye configuration are preserved in the fossil record? It would seem very likely that they are. However, as the developments were occurring in animals with very little in the way of hard tissue (without shells or backbones and with cartilaginous skulls), such fossils are unlikely to be well preserved. Perhaps, most 
importantly though, because the developments involved subtle changes in soft tissues, it would appear that enormous good fortune will be needed to discover fossils with eyes that could be interpreted in terms of transitions toward the vertebrate-style eye. Finding fossil signs of the development of an intraocular lens and of extraocular muscles would seem a realistic possibility; see for example Young (2008). But finding fossil evidence for the gradual invagination of the retina to form an eye-cup would seem to require much more luck than one could reasonably hope for.

Nevertheless, many clues to the numerous gradations that occurred during eye evolution are retained in surviving organisms in the morphology and biochemistry of their retinal cells and, perhaps most importantly, in their genes.

Gradations in the Morphology of Ciliary Photoreceptors

One example of surviving clues is provided by the morphology of photoreceptor cells across chordate taxa (see Lamb et al. 2007, Fig. 2). The photoreceptors in all these taxa are very closely related, being ciliary in nature, with light-sensitive membranes protruding from a modified cilium, and with synaptic output occurring at a terminal not very far from the cell body (soma). But a distinct gradation is apparent in the structure of these ciliary photoreceptors from larval sea-squirts (ascidia), through hagfish, to lampreys, and then to jawed vertebrates - a sequence corresponding to the stages of antiquity at which those other lines diverged from the vertebrate line. In ascidian photoreceptors, the light-sensitive membranes of the outer segment are arranged more-or-less longitudinally, rather like the petals of a rosebud, but in hagfish outer segments, the membranes are splayed out laterally from a central cilium. In the lamprey retina, these membranes are quite neatly stacked, with the cilium at one side. Finally, in jawed vertebrates, the membrane stacking is exquisitely organized in both cones and rods. In rods, a further organizational arrangement occurs whereby the openings between the stacked membranes seal over, creating free-floating discs that are surrounded by an outer (plasma) membrane. At the other end of the photoreceptor, at its synaptic output terminal, a similar gradation exists across these organisms, from a simple synaptic density in hagfish photoreceptors to a very specialized synaptic ribbon structure in jawed vertebrates.

\section{Gradations in the Opsin Photopigments}

As one example of the wealth of clues to vertebrate eye evolution that are contained in the genes of organisms, we now briefly discuss the genes for visual pigments (opsins). A great deal of information relevant to evolution is

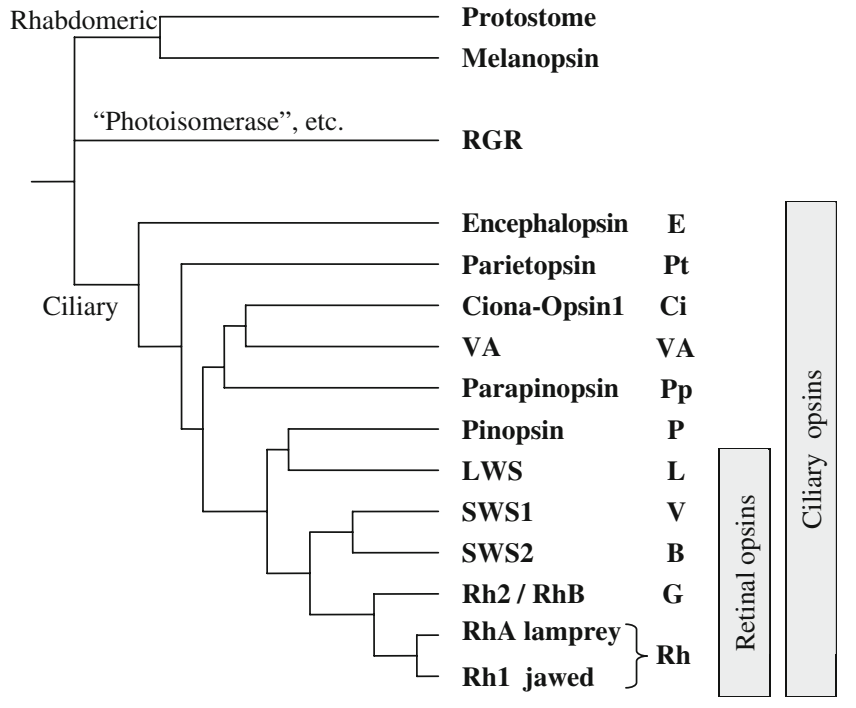

Fig. 5 The evolution of vertebrate opsins. The dendrogram shows the relationship between the classes of opsins found not only in our own retinal photoreceptors (retinal opsins) but also in other light-sensing regions; e.g., in the pineal (pinopsin) and elsewhere (encephalopsin) in vertebrates, as well as in the light-sensitive regions of other organisms (e.g., Ciona opsin, and the rhabdomeric opsins of most invertebrates). The primordial opsin had already diverged into three classes (rhabdomeric, "photoisomerase-like," and ciliary opsins) before protostomes (including most invertebrates) and deuterostome (including vertebrates) diverged, at least 580 Mya. Within the retina of vertebrates, the ciliary opsin diverged into LWS and short-wavelength sensitive branches, with the latter splitting twice more, to give two "SWS" and two "Rh" groups. Originally, all five of these opsins were utilized in cones, and it was not until after the ancestors of lampreys and jawed vertebrates separated (about 500 Mya) that vertebrate rhodopsin (Rh1) became a distinctively "rod opsin" and that the other distinguishing features of rods evolved [From Lamb et al. (2007) Fig. 3; first published in Nature Reviews Neuroscience 8, 2007 (C) Nature Publishing Group, a division of Macmillan Publishers Limited]

contained within the genes of a single species, but vastly more information is available through comparison of genes across species. Analysis of the closeness (or distance) of the individual gene sequences to each other permits the generation of evolutionary branching patterns known as dendrograms, such as that illustrated in Fig. 5. Such diagrams document the relationships between opsins across taxa and suggest the sequence in which the opsins in different tissues and in different organisms may have evolved.

By the time of the divergence of protostome and deuterostome organisms, about 580 Mya, a primordial opsin had already diversified into three classes that are referred to as rhabdomeric, photoisomerase-like, and ciliary; all three classes have been shown to coexist in a wide variety of invertebrate and vertebrate species.

The ciliary opsins are so called because they are expressed in ciliary photoreceptor cells. In vertebrates, such photopigments include not only rhodopsin and the cone opsins in retinal photoreceptors and the closely related 
opsins in pineal and parapineal photoreceptors (at least in non-mammals), but they also include encephalopsin (originally thought to be expressed in deep brain receptors) and a number of "orphan" opsins for which the function and location remain mysterious. Other retinal cells contain additional ciliary opsins - for example, vertebrate ancient (VA) in horizontal cells and amacrine cells of fish - though it is not entirely clear that these cells are of ciliary origin.

According to the analysis of relatedness in Fig. 5, the ciliary class of opsins has branched many times, with encephalopsin having diverged at a very early stage from the line that gave rise to retinal opsins. The single ciliary opsin in the ascidian Ciona (in the larval tadpole-like stage of this sea-squirt) appears to have diverged before the vertebrate retinal opsins began diverging from each other. During the evolution of the vertebrate retina, a number of branchings occurred: A primordial retinal opsin branched to form a long-wave sensitive (LWS) opsin and a short-wave (ultraviolet) sensitive opsin, the latter of which in turn branched several times to produce opsins sensitive (typically) in the violet, blue, and green regions of the visible spectrum. These four classes of cone pigment had evolved before the time that the forerunner of lampreys had diverged from the line that became the jawed vertebrates, around 500 Mya. True rhodopsin (Rh1, expressed in the rods of jawed vertebrates) arose more recently, after the divergence of lampreys. Nevertheless, there is a closely related opsin (RhA) present in one class of lamprey photoreceptors; these cells are presumed not to have achieved the single-quantum detection capability of vertebrate rods, though this remains to be tested.

In any case, the subtle differences in opsin sequences between species, in conjunction with the good correlation that exists between the deduced opsin branching patterns and the presumed branching patterns between species, are entirely consistent with the notion that a myriad of tiny changes have occurred in opsin sequences over the eons, and that those that have had advantages under different conditions have survived in different guises in different cells.

Melanopsin Finally, we note the remarkable evolutionary position of one opsin, melanopsin. This molecule is clearly a member of the family of rhabdomeric opsins that, until recently, had been thought to be expressed only in the rhabdomeric photoreceptors of invertebrates; it is only distantly related to the other (ciliary) opsins found in the vertebrate retina. Yet melanopsin is expressed in a class of mammalian retinal ganglion cells centrally involved in the regulation of the circadian cycle and in other "non-imaging" actions of light. This (and other evidence) led to the proposal that mammalian retinal ganglion cells have their origins in the same kind of primordial rhabdomeric photoreceptor cell that still exists today and that represents the primary detector of light in the eyes of flies, squid, and other protostomes (Arendt 2003). Thus, as sketched in Fig. $1 \mathrm{~b}$ and c, it is possible to think of our retinal ganglion cells as the descendants of rhabdomeric photoreceptors that lost their rhabdomeric membranes but (in some cases) retained their rhabdomeric pigment, and that evolved to receive synaptic input from the ciliary photoreceptor pathway.

Transduction Pathways and Transcription Factors Two other topics relating genes to eye evolution are beyond the scope of this article. These involve analysis of the information inherent in gene families relating to (1) the evolution of the sensory signaling pathways (e.g., phototransduction, adaptation, recovery, and signal transmission) in photoreceptors and other retinal neurons and (2) the evolution of transcription factors and other genes involved in specifying the differentiation, patterning, and development of the eye. Analysis of these gene families in the future seems certain to provide major new information about the sequence of gradual changes that took place in the evolution of our eyes.

\section{Future Tests Based on Predictions of the Model}

An important aspect of the scenario that we have sketched in Fig. 1 and described above is that it leads to a number of explicit predictions, which may be examined experimentally and thereby used to test the accuracy of the model. We now list a number of the predictions, and we describe some of the experiments that could be applied to test them.

\section{Phototransduction homologies}

Our model predicts not only that the ciliary photoreceptors of all chordates should exhibit close homology to each other but that molecular analysis of the transduction components should show evidence for the phylogenetic sequence: tunicates (e.g., sea-squirts), hagfish, lampreys, and jawed vertebrates. To test these predictions, one could identify all the molecular components of the phototransduction cascade (opsin, G-protein, phosphodiesterase, cyclic nucleotide-gated channel, arrestin, etc.) across the range of taxa: e.g., in the tadpole-like larva of the seasquirt Ciona intestinalis (an ascidian), in hagfish, and in lampreys. One could also record the properties of the electrical responses of the cells to light, using either massed potential recordings or single-cell recordings. The molecular components and the electrical responses could then be compared with previously published data for the photoreceptors of jawed vertebrates to test for evidence of homologies and for any phylogenetic relationships. This 
comparison would be greatly assisted if the entire genome of a hagfish species were available, but as yet, this has not been reported.

\section{Ciliary to rhabdomeric photoreceptor contact}

Our model requires that at an early stage in chordate evolution there should have been synaptic contact from ciliary photoreceptors onto rhabdomeric photoreceptors. It might therefore be possible to locate such contacts between the two classes of photoreceptor in extant protochordates, such as Amphioxus and C. intestinalis. There might also be microvillar membranes remaining in the retinal projection neurons (ganglion cells) of extant chordates. Such findings would lend great weight to the model, though the search may be comparable to finding a needle in a haystack.

\section{The hagfish retina should lack bipolar cells}

Our model predicts that the hagfish retina should not contain bipolar cells and that the photoreceptors should synapse directly onto projection neurons (ganglion cells). To test this prediction, one could attempt to immunolabel the hagfish retina using established markers for retinal bipolar cells from the vertebrate retina. If bipolar cells were identified in the hagfish retina and were found to connect photoreceptors with ganglion cells, then our model would either fall or require major revision. To test for direct contact between photoreceptors and ganglion cells, one could also employ retrograde labeling of the optic nerve, to clearly identify projection neurons, and then examine the synaptic input(s) to these cells at an ultrastructural level. One could also use the recent "gene gun" technology and the classical Golgi approach to label individual cells in the hagfish retina so as to allow detailed examination of their connectivity and to establish the number of neuronal elements in the retinal pathway.

\section{The lamprey retina should lack true rods}

Based on the ideas of other studies as well as our own, rod photoreceptors did not evolve until after the divergence of lampreys from jawed vertebrates, and therefore the photoreceptors of the lamprey retina should not exhibit the crucial features that characterize vertebrate rods: e.g., anatomical features such as sealed-off disc membranes, and functional features such as the reliable detection of single-photon responses, together with extreme stability of the visual pigment molecule against spontaneous thermal activation (i.e., a very low "dark noise"). We propose that experiments be undertaken examining the ultrastructure of the outer segment and characterizing the electrical responses at the single-cell level to test these predictions.
5. The lamprey retina should lack the rod bipolar cell pathway

Although the lamprey retina possesses bipolar cells that are likely to be homologous to the cone bipolar cells of the jawed vertebrate retina, we expect that it should not contain rod bipolar cells (as it should not contain "true" rods) nor should it contain the special AII amacrine cells that, in the jawed vertebrate retina, feed the rod-driven signals into the conedriven pathway at the level of the inner retina. These predictions could be tested by immunohistochemical labeling, employing markers for rod bipolar cells and for AII amacrine cells previously used successfully on other vertebrate retinae. In addition, electrophysiological recordings could be made within the inner retina in a search for cells with the characteristic electrical responses of rod bipolar cells.

6. Retinal bipolar cells should be homologous to photoreceptors

Our model predicts that retinal bipolar cells arose through some kind of duplication event, either of photoreceptors themselves or of a precursor of photoreceptors (e. g., of a ciliated neuron contacting the CSF). In either case, we would predict that the molecular components of retinal bipolar cells should be very closely related to those of the photoreceptors. A number of similarities have already been identified, but we now propose that a much more comprehensive comparison of the molecular components of the two cell types be undertaken.

We hope that in the near future we and others will be able to put the above predictions to the test. Accordingly, we expect that it will soon be possible to provide additional evidence that could lead to one of a range of outcomes, including support for the model, revision of the model, or rejection of the model.

\section{Summary}

The information that is now available provides powerful clues to the sequence of events that occurred during the evolution of the vertebrate eye. That process involved a vast number of slight improvements to a primordial photoreceptive region, though very few of the transitional steps have survived to the present day, because of competition from animals with eyes that were superior. Furthermore, there are likely to be few signs in the fossil record because the refinements occurred in soft tissue that is rarely preserved. We propose that the "eye" of the hagfish provides a glimpse into a state that arose some $530 \mathrm{Mya}$, in which craniate animals had evolved bilateral non-imaging photoreceptive organs. The subsequent stages of evolution of the optical 
apparatus and information-processing capacity of the retina occurred with remarkable rapidity, within a period of 30 million years, so that by 500 Mya early vertebrates possessed eyes fundamentally similar to our own.

\section{References}

Arendt D. Evolution of eyes and photoreceptor cell types. Int J Dev Biol. 2003;47:563-71.

Arendt D, Wittbrodt J. Reconstructing the eyes of Urbilateria. Philos Trans R Soc Lond B Biol Sci. 2001;356:1545-63. doi:10.1098/ rstb.2001.0971.

Darwin C. On the origin of species by means of natural selection, or the preservation of favoured races in the struggle for life. London: John Murray; 1859.

Dobzhansky T. Nothing in biology makes sense except in the light of evolution. Am Biol Teach. 1973;35:125-9.

Haeckel E. Die Natürliche Schöpfungsgeschichte. Berlin: Georg Reimer; 1868.
Holmberg K. The cyclostome retina. In: Crescitelli F, editor. Handbook of Sensory Physiology, vol VII/5, The Visual System of Vertebrates. Berlin: Springer; 1977. p. 47-66.

Johnson PT, Williams RR, Cusato K, Reese BE. Rods and cones project to the inner plexiform layer during development. J Comp Neurol. 1999;414:1-12. doi:10.1002/(SICI)1096-9861 (19991108)414:1<1::AID-CNE1>3.0.CO;2-G.

Lamb TD, Pugh EN Jr. Dark adaptation and the retinoid cycle of vision. Prog Retin Eye Res. 2004;23:307-80. doi:10.1016/j. preteyeres.2004.03.001.

Lamb TD, Collin SP, Pugh EN Jr. Evolution of the vertebrate eye: Opsins, photoreceptors, retina, and eye-cup. Nat Rev Neurosci. 2007;8:960-75. doi:10.1038/nrn2283.

Locket NA, Jorgensen JM. The eyes of hagfishes. In: Jorgensen JM, Lomholt JP, Weber RE, Malte H, editors. The Biology of Hagfishes, Chap. 34. London: Chapman and Hall; 1998. p. 541-6.

Richards RJ. von Baer, Karl Ernst (1792-1876), and Haeckel, Ernst (1834-1919). In: Ruse M, Travis J, editors. Evolution: The First Four Billion Years. Cambridge: Harvard University Press; 2009.

von Baer KE. Entwickelungsgeschichte der Thiere: Beobachtung und Reflexion. Königsberg: Bornträger; 1828.

Young GC. Early evolution of the vertebrate eye: fossil evidence. Evo Edu Outreach. 2008;1(4). doi:10.1007/s12052-008-0087-y. 